\title{
Effect of acute hyperglycaemia on gall bladder contraction induced by cholecystokinin in humans
}

\author{
S Y de Boer, A A M Masclee, M C W Jebbink, J Schipper, H H P J Lemkes, J B M J Jansen, \\ C B H W Lamers
}

\begin{abstract}
This study examined the effect of acute hyperglycaemia, induced by intravenous glucose, on gall bladder motility. Six healthy volunteers were studied in random order on three occasions during normoglycaemia and hyperglycaemia with blood glucose concentrations stabilised at 8 and $15 \mathrm{mmol} / \mathrm{l}$. Gall bladder volumes, measured with ultrasonography, were studied before and during infusion of stepwise increasing doses of cholecystokinin (CCK-33; 0.25, 0.5, and 1.0 IDU.kg-1. $\mathbf{h}^{-1}$ ). Each dose was given for $\mathbf{3 0}$ minutes. Pancreatic polypeptide (PP) secretion was determined as an indirect measure of cholinergic tone. Infusion of CCK-33 resulted in significant dose dependent reductions in gall bladder volume in all three experiments. Compared with normoglycaemia the gall bladder contraction was significantly $(p<0.05)$ reduced during infusion of 0.25 and $0.5 \mathrm{IDU} \mathrm{kg}^{-1} \cdot \mathrm{h}^{-1} \mathrm{CCK}-33$ in the $8 \mathrm{mmol} / \mathrm{l}$ hyperglycaemic experiment, and during infusion of $0.25,0.5$, and $1.0 \mathrm{IDU}$ $\mathrm{kg}^{-1} \cdot \mathrm{h}^{-1}$ CCK-33 in the $15 \mathrm{mmol}$ hyperglycaemic experiment. During hyperglycaemia basal plasma PP concentrations and PP secretion in response to $\mathrm{CCK}-33$ were significantly $(p<0.05)$ reduced. It is concluded that blood glucose concentrations affect gall bladder motility, that an acute hyperglycaemia at 8 and $15 \mathrm{mmol} / \mathrm{l}$ reduces the gall bladder responsiveness to CCK-33 in a dose dependent manner, and that hyperglycaemia reduces basal and CCK-33 stimulated plasma PP concentrations, suggesting impaired cholinergic activity during hyperglycaemia.

(Gut 1993; 34: 1128-1132)
\end{abstract}

Department of Gastroenterology-

Hepatology,

$S$ Y de Boer

A A M Masclee

M C W Jebbink

J B M J Jansen

C B H W Lamers

Department of

Radiology,

J Schipper

Department of

Endocrinology,

University Hospital

Leiden, The Netherlands

H H P J Lemkes

Correspondence to:

A A M Masclee, Department

of Gastroenterology-

of Gastroenterology-

Hepatology, University

Leiden, The Netherlands.

Accepted for publication

8 December 1992
Recently several studies have emphasised the importance of serum glucose concentrations in the regulation of gastrointestinal function. ${ }^{1-6}$ In healthy subjects hyperglycaemia induced by intravenous infusion of glucose reduces gastric emptying of solid and liquid meals, ${ }^{1-3}$ gastric acid secretion, ${ }^{45}$ and pancreatic secretion. ${ }^{46}$ Little is known, however, about the effect of hyperglycaemia on gall bladder motility. It has been suggested that hyperglycaemia reduces gastrointestinal function through inhibition of the vagal cholinergic system. ${ }^{23}$

This study was undertaken to investigate the influence of hyperglycaemia on gall bladder motility. Gall bladder contraction in response to infusion of graded doses of cholecystokinin (CCK-33) was measured during normoglycaemia and during acute hyperglycaemia with blood glucose concentrations stabilised at 8 and $15 \mathrm{mmol} / \mathrm{l}$. A second aim of the study was to examine the tone of the cholinergic system during hyperglycaemia. This was accomplished indirectly by measuring pancreatic polypeptide (PP) secretion, which is known to be dependent on cholinergic tone and reduced during hyperglycaemia in healthy subjects. ${ }^{78}$

\section{Materials and methods}

\section{SUBJECTS}

Six healthy young men (aged 24-32) participated in the study. None was taking chronic medication or had a history of gastrointestinal disease, surgery, or diabetes mellitus. Informed consent was obtained from each person and the protocol was approved by the ethics committee of Leiden University Hospital.

\section{EQUIPMENT}

Gall bladder volumes, measured by real time ultrasonography (Technicare, $3.5 \mathrm{MHz}$ transducer), were calculated by the sum of cylinders method with a computerised system. ${ }^{910}$ In this method the longitudinal scan of the gall bladder is divided into series of cylinders of equal height, with diameters perpendicular to the longitudinal axis of the gall bladder image. The uncorrected volume is the sum of volumes of these separate cylinders. To correct for the displacement of the longitudinal image of the gall bladder from the central axis, a correction factor is calculated from the longitudinal and transversal scans of the gall bladder. Gall bladder volume is calculated by multiplication of the uncorrected volume by the square of the correction factor. The mean of two measurements was used for further analysis. The assumptions and the mathematical formula used to calculate gall bladder volume have been described and validated previously by Everson et al and Hopman et al. ${ }^{10}$

\section{TEST PROCEDURE}

Each subject participated in three experiments performed in random order, with an interval of at least seven days between each. In the three experiments the subjects were either normoglycaemic or hyperglycaemic with blood glucose concentrations stabilised at 8 or $15 \mathrm{mmol} / \mathrm{l}$. All experiments were started at 0900 after an overnight fast. An intravenous cannula was inserted into an antecubital vein of one arm for blood sampling. A second intravenous line was positioned in the antecubital vein of the other arm for infusions of $20 \%$ glucose and $0.9 \%$ saline. An acute hyperglycaemia at 8 or $15 \mathrm{mmol} / \mathrm{l}$ was obtained with a modified glucose clamp tech- 


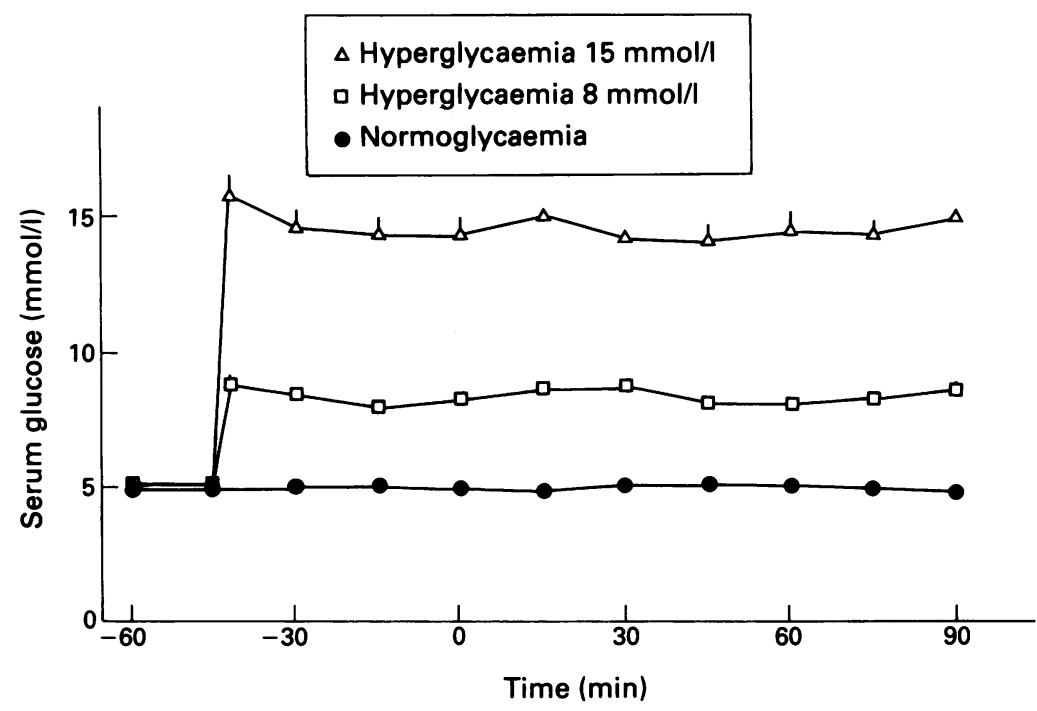

Figure 1: Serum glucose concentrations (mmolll, mean (SEM)) in six healthy subjects during normoglycaemia and hyperglycaemic clamping.

nique. ${ }^{112}$ The acute rise in blood glucose concentrations was induced by an intravenous bolus injection of a glucose $20 \%$ solution 45 minutes before the start of CCK-33 infusion. The amount of glucose given was calculated from body weight. After a bolus injection the rate of glucose infusion was adjusted to maintain a blood glucose concentration of about 8 or $15 \mathrm{mmol} / \mathrm{l}$ according to the protocol. Serum glucose concentrations were measured every 2.5 to five minutes by the glucose oxidase method (Beckman glucose analyser, Beckman Instruments).

At zero minutes a continuous intravenous infusion of CCK-33 (Ferring, Malmo, Sweden) was started. Cholecystokinin was given in stepwise increasing doses of $0 \cdot 25 \mathrm{IDU} \mathrm{kg}^{-1} \cdot \mathrm{h}^{-1}, 0 \cdot 5$ IDU $\mathrm{kg}^{-1} \cdot \mathrm{h}^{-1}$, and $1 \cdot 0$ IDU $\mathrm{kg}^{-1} \cdot \mathrm{h}^{-1}$. Each dose was for 30 minutes. Previous studies have shown that during infusions of $0.25,0.5$, and 1.0 IDU $\mathrm{kg}^{-1} \cdot \mathrm{h}^{-1}$ plasma cholecystokinin (CCK) concentrations correspond with plasma CCK

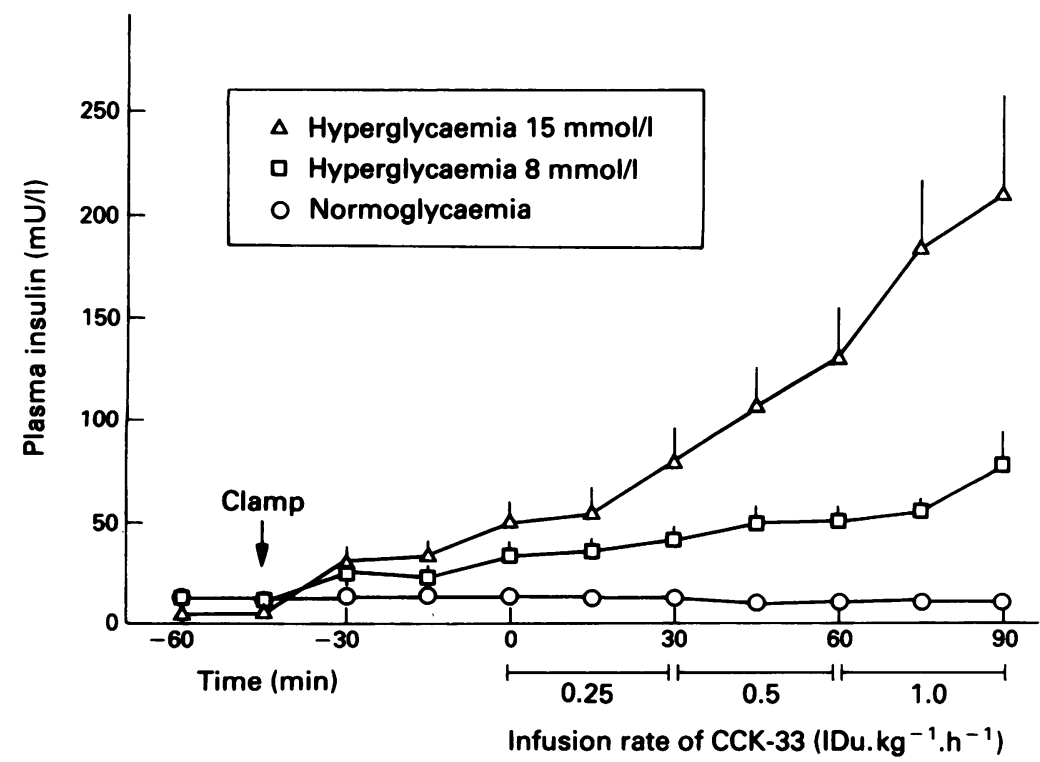

Figure 2: Plasma insulin concentrations ( $m U / l$, mean (SEM)) during normoglycaemia and hyperglycaemic clamping. concentrations measured after ingestion of a light, normal, and fatty meal. ${ }^{13}$ Gall bladder images were taken in duplicate every 15 minutes at $-60,-45,-30,-15,0,15,30,45,60,75$, and 90 minutes. Blood samples for measurement of PP, CCK, and insulin were drawn each time a gall bladder image was taken. Plasma PP concentrations were measured by a sensitive and specific radioimmunoassay as described previously. ${ }^{14}$ Plasma CCK was measured by a sensitive and specific radioimmunoassay with antibody T204. ${ }^{1516}$ This antibody binds to all carboxy terminal CCK peptides containing the sulphated tryosyl region. The detection limit of the assay was $0.5 \mathrm{pmol} / 1$ plasma. The within assay variation ranged from $4 \cdot 6 \%$ to $11 \cdot 5 \%$ and the between assay variation from $11 \cdot 3 \%$ to $26 \cdot 1 \% .{ }^{15}$ Plasma insulin was measured by radioimmunoassay as described previously. ${ }^{17}$

\section{STATISTICAL ANALYSIS}

Results are expressed as mean (SEM). The integrated incremental PP secretion in response to CCK-33 infusion was determined by calculating the area under the plasma concentration-time curve after subtraction of the basal value. Gall bladder volumes are expressed in $\mathrm{cm}^{3}$ or as percentages, considering the gall bladder volume at the start of the infusion of CCK-33 as $100 \%$ (zero minutes). Data were analysed for the significance of differences by analysis of variance (ANOVA). When this indicated a probability of less than 0.05 for the null hypothesis StudentNewman-Keuls analyses were performed to determine which values differed significantly. The significance level was set at $\mathrm{p}<0.05$.

\section{Results}

SERUM GLUCOSE CONCENTRATIONS

Fasting serum glucose concentrations in the normo and hyperglycaemic experiments were $5 \cdot 1(0 \cdot 3) \mathrm{mmol} / \mathrm{l}, 5 \cdot 0(0 \cdot 7) \mathrm{mmol} / 1$, and $5 \cdot 0(0.3)$ $\mathrm{mmol} / \mathrm{l}$ respectively. Figure 1 shows mean serum glucose concentrations reached during clamping.

\section{PLASMA INSULIN CONCENTRATIONS}

Fasting plasma insulin concentrations were not significantly different between the three experiments (Fig 2). In the normoglycaemic experiment no significant changes in plasma insulin were found during infusion of CCK-33. After initiating the hyperglycaemic clamp at -45 minutes, plasma insulin concentrations increased significantly $(\mathrm{p}<0.05)$ starting from -30 minutes in the 8 and $15 \mathrm{mmol} / \mathrm{l}$ glucose clamps. At the end of the $15 \mathrm{mmol} / \mathrm{l}$ glucose clamp plasma insulin concentrations of 212 (45) $\mathrm{mU} / \mathrm{l}$ were reached.

\section{GALL BLADDER VOLUMES}

Basal gall bladder volumes were not significantly different between the three experiments $(28(8)$ $\mathrm{cm}^{3}$ (normoglycaemia), $29(9) \mathrm{cm}^{3}(8 \mathrm{mmol} / \mathrm{l}$ hyperglycaemia), and $29(9) \mathrm{cm}^{3}(15 \mathrm{mmol} / \mathrm{l}$ 


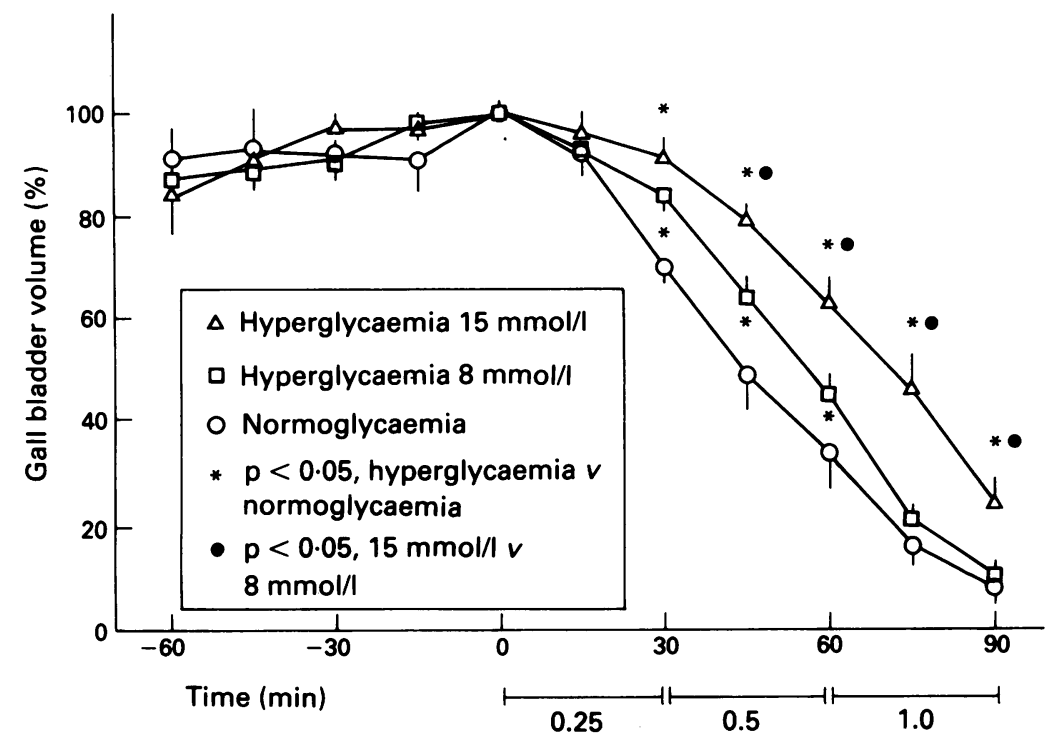

Infusion rate of CCK-33 (IDU. $\left.\mathrm{kg}^{-1} \cdot \mathrm{h}^{-1}\right)$

Figure 3: Gall bladder volumes (\%, mean (SEM)) during normoglycaemia and hyperglycaemia clamping. From 0 to 90 minutes gall bladder volumes were in response to cholecystokinin (CCK-33) infusion.

hyperglycaemia)). Infusion of $0 \cdot 25,0 \cdot 5$, and $1 \cdot 0$ IDU $\mathrm{kg}^{-1} \cdot \mathrm{h}^{-1}$ CCK-33 resulted in significant reductions in gall bladder volumes from $100 \%$ to $70 \%(3 \%)(\mathrm{p}<0.05), 34 \%(7 \%)(\mathrm{p}<0.05)$, and $8 \%(3 \%)(\mathrm{p}<0.05)$ during normoglycaemia, from $100 \%$ to $84 \%(3 \%)(\mathrm{p}<0.05), 45 \%(4 \%)$ $(\mathrm{p}<0.05)$, and $10 \%(2 \%)(\mathrm{p}<0.05)$ during the $8 \mathrm{mmol} / \mathrm{l}$ glucose clamp, and from $100 \%$ to $91 \%$ (4\%) $(\mathrm{p}<0.05), 63 \%(5 \%)(\mathrm{p}<0.05)$, and $24 \%$ $(5 \%)(\mathrm{p}<0.05)$ during the $15 \mathrm{mmol} / \mathrm{l}$ glucose clamp (Fig 3). During the $15 \mathrm{mmol} / 1$ hyperglycaemia gall bladder contraction was significantly reduced $(\mathrm{p}<0.05)$ from 30 to 90 minutes compared with normoglycaemia and from 45 to 90 minutes compared with the $8 \mathrm{mmol} / \mathrm{l}$ glucose clamp experiment. During the $8 \mathrm{mmol} / \mathrm{l}$ hyperglycaemia gall bladder contraction was significantly reduced at 30,45 , and 60 minutes compared with normoglycaemia.

\section{PLASMA CCK CONCENTRATIONS}

Fasting plasma CCK concentrations were not significantly different between the three experiments (Table). Infusion of $0.25,0.5$, and 1.0 IDU $\mathrm{kg}^{-1} \cdot \mathrm{h}^{-1} \mathrm{CCK}-33$ resulted in significant $(\mathrm{p}<0.05)$ increases in plasma CCK concentrations over baseline in all experiments. The plasma CCK concentrations in response to infusion of graded doses of CCK-33 were not significantly different between the three experiments.

Plasma cholecystokinin (CCK) concentrations (pmol/l, mean (SEM)) before and during infusion of stepwise increasing doses of CCK-33

\begin{tabular}{lccc}
\hline $\begin{array}{l}\text { CCK-33 infusion rate } \\
\left(I D U \mathrm{~kg}^{-T} . h^{-1}\right)\end{array}$ & Normoglycaemia & $\begin{array}{l}\text { Hyperglycaemia } \\
(8 \mathrm{mmol} / \mathrm{l})\end{array}$ & $\begin{array}{c}\text { Hyperglycaemia } \\
(15 \mathrm{mmol} / \mathrm{l})\end{array}$ \\
\hline Baseline & $3 \cdot 4(0 \cdot 3)$ & $3 \cdot 5(0 \cdot 4)$ & $3 \cdot 5(0 \cdot 3)$ \\
0.25 & $4 \cdot 5(0 \cdot 2)^{\star}$ & $4 \cdot 4(0.3)^{\star}$ & $4 \cdot 5(0 \cdot 2)^{\star}$ \\
0.5 & $6 \cdot 5(0 \cdot 4)^{\star}$ & $6 \cdot 6(0 \cdot 3)^{\star}$ & $5 \cdot 6(0 \cdot 3)^{\star}$ \\
$1 \cdot 0$ & $10 \cdot 9(0 \cdot 7)^{\star}$ & $11 \cdot 0(0 \cdot 8)^{\star}$ & $10 \cdot 2(0 \cdot 6)^{\star}$ \\
\hline
\end{tabular}

${ }^{\star} \mathrm{p}<0.05$ compared with baseline.

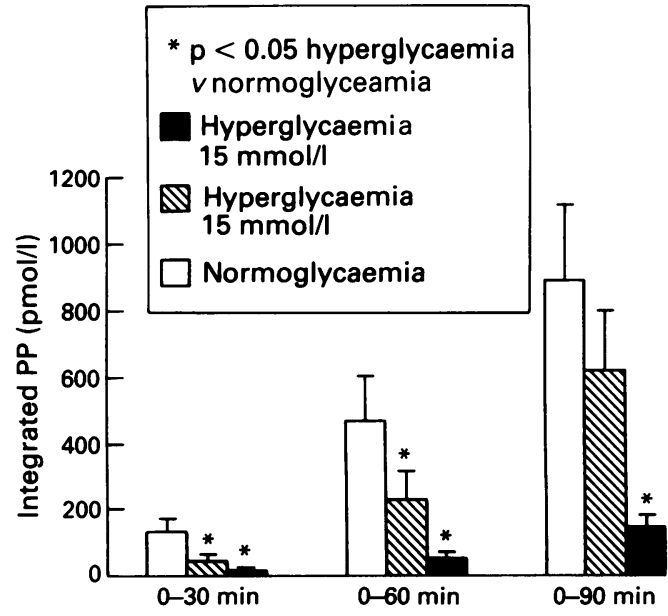

Figure 4: Integrated incremental pancreatic polypeptide (PP) secretion ( $p$ mol/l, mean (SEM) response to cholecystokinin (CCK-33) infusion during normoglycaemia and hyperglycaemic clamping.

PLASMA PANCREATIC POLYPEPTIDE CONCENTRATIONS

Fasting plasma PP concentrations were not significantly different between the three experiments (17 (2) pmol/l (normoglycaemia), 17 (2) pmol/l (8 mmol/l hyperglycaemia), and 19 (2) pmol (15 mmol/l hyperglycaemia)). From -45 to zero minutes no significant changes in PP concentration were found during normoglycaemia whereas after initiating hyperglycaemia, plasma PP concentrations decreased significantly $(\mathrm{p}<0.05)$ both during the 8 and 15 mmol/l glucose clamp. At 0 minutes mean plasma PP concentrations were 17 (2) pmol/l (normoglycaemia), 13 (1) $\mathrm{pmol} / \mathrm{l}$ (8 mmol/l hyperglycaemia), and 12 (1) pmol/l (15 mmol/l hyperglycaemia). Infusion of CCK-33 from $0 \cdot 25$ IDU kg $\mathrm{g}^{-1} \cdot \mathrm{h}^{-1}$ to $1.0 \mathrm{IDU} \mathrm{kg}^{-1} \cdot \mathrm{h}^{-1}$ resulted in significant $(p<0.05)$ increases in plasma PP in the normoglycaemic and $8 \mathrm{mmol} / \mathrm{l}$ hyperglycaemic experiments whereas during the 15 mmol/l hyperglycaemic experiment plasma PP concentrations increased significantly only during infusion of $1 \cdot 0$ IDU kg $\mathrm{g}^{-1} \cdot \mathrm{h}^{-1} \mathrm{CCK}-33$. Compared with normoglycaemia, during the 15 $\mathrm{mmol} / \mathrm{l}$ hyperglycaemia the integrated incremental PP secretion in response to CCK-33 was significantly reduced during infusion of 0.25 , 0.5 , and 1.0 IDU kg ${ }^{-1} \cdot \mathrm{h}^{-1}$ CCK-33 (Fig 4). During the $8 \mathrm{mmol} / \mathrm{l}$ hyperglycaemia the integrated PP secretion in response to CCK-33 was significantly $(\mathrm{p}<0.05)$ reduced compared with normoglycaemia during infusion of 0.25 and 0.5 IDU kg $^{-1} \cdot \mathrm{h}^{-1}$.

\section{Discussion}

The results of this study indicate that in healthy humans, gall bladder motility induced by CCK33 is related to blood glucose concentrations. During an acute hyperglycaemia of 8 and 15 $\mathrm{mmol} / \mathrm{l}$ the gall bladder contraction in response to CCK-33 was inhibited in a dose dependent manner.

In several studies it has been suggested that gastrointestinal function is related to blood glucose concentrations. ${ }^{1-6}$ During an acute 
hyperglycaemia induced by intravenous infusion of glucose, gastric emptying, ${ }^{13}$ gastric acid secretion $^{45}$ and pancreatic enzyme secretion ${ }^{46}$ are reduced in healthy subjects. McGregor et al have shown that dextrose given intravenously inhibits meal stimulated intraduodenal bilirubin output. ${ }^{4}$ It is not known from that study, however, whether the reduction in intraduodenal bilirubin output resulted from hyperglycaemia induced alterations in gastric emptying of the meal, alterations in endogenous CCK secretion, or alterations in the responsiveness of the gall bladder to CCK. Unfortunately, in several of the studies that analysed the effect of intravenous glucose on gastrointestinal function, serum glucose concentrations have not been reported. ${ }^{1346}$ Also, serum glucose concentrations may vary considerably during a constant infusion of glucose due to insulin secretion. To accurately determine the effect of hyperglycaemia on gastrointestinal function we think it is essential to stabilise serum glucose concentrations as in the present study by glucose clamp techniques.

In recent years it has been possible to study the mechanisms involved in the regulation postprandial gall bladder motility in detail because of the development of sensitive and specific radioimmunoassays for measurement of CCK and the availability of ultrasonography and cholescintigraphy as non-invasive methods to measure human gall bladder motility. ${ }^{9151819}$ Two findings point to an important role of CCK in the regulation of postprandial gall bladder motility. Firstly, after eating a meal gall bladder contraction is closely related to plasma CCK concentrations. ${ }^{1820}$ Secondly, intravenous infusion of CCK to plasma concentrations comparable with those found after meals induces similar gall bladder contraction. ${ }^{131819}$ By stimulating gall bladder contraction in our study through infusion of CCK -33 instead of meal ingestion we precluded the influences of alterations in gastric emptying and endogenous secretion of CCK on gall bladder motility induced by hyperglycaemia. Infusion of stepwise increasing doses of CCK-33 in our experiments resulted in dose dependent increments in plasma CCK, corresponding to plasma CCK concentrations found after ingestion of a light, normal, and fatty meal. ${ }^{18} 20$ Plasma CCK concentrations reached during infusion of CCK-33 were in the same range in the three experiments, thus excluding the possibility that differences in gall bladder contraction between the hyperglycaemic and normoglycaemic experiments are related to differences in plasma CCK concentrations.

The mechanism responsible for the inhibitory action of hyperglycaemia on gall bladder motility is not known. It is possible that metabolic, hormonal, or neural changes secondary to hyperglycaemia, rather than glucose itself, modulate gastrointestinal function. For instance, high glucose concentrations stimulate insulin secretion in healthy volunteers and hyperinsulinemia affects gastrointestinal function. Previous studies have shown, however, that insulin exerts a stimulatory, rather than an inhibitory effect on intestinal motility. ${ }^{21}{ }^{22}$ Furthermore, in type I diabetic patients, hyperglycaemia reduces gastric emptying indicating that insulin is not responsible for the inhibitory effect of hyperglycaemia on gastrointestinal function. ${ }^{23}$

Several arguments are in favour of an inhibitory effect of hyperglycaemia on gall bladder motility by suppressing activity of the vagal cholinergic system. Firstly, the effect of CCK on the gall bladder in vivo is dependent on vagal cholinergic activity. Cholinergic blockade with atropine reduces contractile responses of the gall bladder to $\mathrm{CCK},{ }^{24}$ and action similar to the effect of hyperglycaemia on gall bladder contraction induced by CCK. Secondly, it has been shown in animals that infusion of glucose suppresses vagal nerve activity. ${ }^{25}$ Thirdly, in our study hyperglycaemia significantly reduced basal and CCK-33 stimulated PP concentrations. The release of PP from the pancreas is known to be under cholinergic control. ${ }^{7}$ Although CCK is a physiological stimulus of PP secretion in humans the effect of CCK on PP secretion is abolished by atropine, indicating that the cholinergic system regulates CCK induced PP secretion. ${ }^{726}$ Fourthly, hyperglycaemia is known to suppress PP secretion through inhibition of cholinergic activity as the inhibitory effect of hyperglycaemia on PP secretion is blunted after truncal vagotomy. ${ }^{27}$ Based on these findings it is postulated that hyperglycaemia affects gall bladder motility, at least in part, through inhibition of cholinergic activity.

It is not known whether in patients with diabetes mellitus, as in healthy subjects, the gall bladder sensitivity to $\mathrm{CCK}$ is influenced by serum glucose concentrations. A reduced gall bladder emptying in response to a meal and to infusion of CCK-33 has been reported in such patients. ${ }^{28}$ This has been associated with the presence of autonomic neuropathy as in diabetic patients without neuropathy the gall bladder contraction in response to CCK is not different from that in healthy subjects. No significant correlation has been found between impaired gall bladder emptying and overall diabetic control as determined by the percentage of total haemoglobin present as glycated haemoglobin. ${ }^{28}$ It remains to be established whether in diabetic patients gall bladder motility is dependent on actual serum glucose concentrations.

In conclusion blood glucose concentrations affect gall bladder motility in healthy subjects, an acute hyperglycaemia at 8 and $15 \mathrm{mmol} / \mathrm{l}$ dose dependently reduces the gall bladder sensitivity to CCK-33, and hyperglycaemia reduces basal and CCK-33 stimulated plasma PP concentrations, suggesting impaired cholinergic activity during hyperglycaemia.

1 McGregor IL, Gueller R, Watts HD, Meyer JH. The effect of acute hyperglycemia on gastric emptying in man. Gastroenterology 1976; 70: 190-6.

2 Barnett JL, Owyang C. Serum glucose concentration as a modulator of interdigestive gastric motility. Gastroenterology 1988; 94: 739-44.

3 Oster-Jorgensen E, Pedersen SA, Larsen ML. The influence of induced hyperglycemia on gastric emptying in healthy
humans. Scand $\mathcal{F}$ Clin Lab Invest 1990; 50:831-6.

4 McGregor IL, Deveney C, Way LW, Meyer JH. The effect of acute hyperglycemia on meal-stimulated gastric, biliary, and pancreatic secretion, and serum gastrin. Gastroenterology

5 Loud FB, Holst JJ, Rehfeld JF, Christiansen J. Inhibition of 
gastric acid secretion in humans by glucagon during euglycemia, hyperglycemia, and hypoglycemia. $\mathrm{Dig} \mathrm{Dis} S \mathrm{C}$ 1988; 33: 530-4.

6 Niederau C, Sonnenberg A, Erckenbrecht J. Effects of intravenous infusion of amino acids, fat or glucose on unstimulated pancreatic secretion in healthy humans. Dig Dis Sci 1985; 30: 445-55.

7 Schwartz TW. Pancreatic polypeptide: a hormone under vagal control. Gastroenterology 1983; 85: 1411-25.

8 Marco J, Hedo JA, Castillo-Olivares J, Villanueva ML. Human pancreatic polypeptide secretion in conditions of exogenous and endogenous hyperglycemia. Diabetologia exogenous and end

9 Everson GT, Braverman DJ, Johnson ML, Kern F. A critical evaluation of real-time ultrasonography for the study of gallbladder volume and contraction. Gastroenterology 1980 79: $40-6$.

10 Hopman WPM, Brouwer WFM, Rosenbusch G, et al. A computerized method for rapid quantification of gallbladder volume from real-time sonograms. Radiology 1985; 154: 236-7.

11 Ward WK, Halter JB, Beard JC, Potr D. Adaptation of B and A cell function during prolonged glucose infusion in human subjects. Am 7 Physiol 1984; 246: E405-11.

12 DeFronzo RA, Tobin JD, Andres R. Glucose clamp technique for quantifying insulin secretion and resistance. $A m \mathcal{F}$ for quantifying insulin secre

13 Hopman WPM, Kerstens PJSM, Jansen JBMJ, Rosenbusch $\mathrm{J}$, Lamers CBHW. Effect of graded physiologic doses of
cholecystokinin on gallbladder contraction measured by cholecystokinin on gallbladder contraction measu

14 Lamers CBHW, Diemel JM, van Leer E, van Leusen R, Peetoom J. Mechanism of elevated serum pancreatic polypeptide concentration in chronic renal failure. $f$ Clin Endocrinol Metab 1982; 55: 922-6.

15 Jansen JBMJ, Lamers CBHW. Radioimmunoassay of cholecystokinin in human tissue and plasma. Clin Chim Acto 1983; 131: 305-16.

16 Jansen JBMJ, Lamers CBHW. Molecular forms of cholecystokinin in plasma from normal and gastrectomized human subjects following a fat meal. Peptides 1987; 8: 801-5.

17 Nijs HGT, Radder JK, Fröhlich M, Krans HMJ. In vivo relation between insulin clearance and insulin action in healthy subjects and insulin-dependent diabetic patients Diabetes 1990; 39: 333-9. 18 Wiener I, Inoue K, Fagan CJ, Lilja P, Watson LC, Thompson
JC. Release of cholecystokinin in man: correlation of blood levels with gallbladder contraction. Ann Surg 1981; 194: 321-7.

19 Masclee AAM, Hopman WPM, Corstens FHM, Rosenbusch G, Jansen JBMI, Lamers CBHW. Simultaneous measurement of gallader emptying with cholescintigraphy and (1) ultrasonography during infusion of physiologic doses of cholecystokinin: a comparison. Radiology 1989; 173:

$20.407-10$

Lopman WPM, Rosenbusch G, Jansen JBMJ, de Jong AJL,
Lamers CBHW. Gallbladder contraction: effects of fatty Lamers CBHW. Gallbladder contraction: effects of fatty

21 Bueno L, Ruckebusch $M$. Insulin and jejunal electrical activity in dog and sheep. Am $\mathcal{F}$ Physiol 1976; 230: 1538-44.

22 Prasad KR, Sarna SK. The central and periferal effects of insulin on migrating myoelectric complexes [Abstract] Gastroenterology 1986; 90: 1589.

23 Fraser RJ, Horowitz M, Maddox AF, Harving PE, Chatterton BE, Dent J. Hyperglycemia slows gastric emptying in type I (insulin-dependent) diabetes mellitus. Diabetologia 1990; 33: $675-80$.

24 Hopman WPM, Jansen JBMJ, Rosenbusch G, Lamers CBHW. Role of cholecystokinin and the cholinergic system in intestinal stimulation of gallbladder contraction in man. in intestinal stimulation of

25 Hirano T Niijima A. Effects of 2-deoxy-D-glucose, glucose and insulin on efferent activity in gastric vagus nerve. Experientia 1980; 36: 1197-8.

26 Meier R, Hildebrand P, Thumshirn M, et al. Effect of loxiglumide, a cholecystokinin antagonist, on pancreatic polypeptide release in humans. Gastroenterology 1990; 99 . $1757-62$.

27 Tsuda K, Seino Y, Mori K, et al. Effect of truncal vagotomy on pancreatic polypeptide response after intravenous glucose administration. Regul Pept 1981; 1: 347-52.

28 Stone BG, Gavaler JS, Belle SH, et al. Impairment of gallbladder emptying in diabetes mellitus. Gastroenterology
1988; 95: 170-6. 